\title{
Integrated Differential Transformer on a Single Printed Circuit Board
}

\author{
Marc Berger, Anne Zygmanowski, Stefan Zimmermann \\ Leibniz University Hannover, Institute of Electrical Engineering and Measurement Technology, \\ Appelstr. 9A, 30167 Hannover, Germany, \\ berger@geml.uni-hannover.de
}

\begin{abstract}
Summary:
The electrical and dielectric properties of samples can be used for various sensing tasks. For specific applications, e.g. continuous in-line measurement of the blood conductivity as a measure of sodium concentration in the blood during dialysis treatment or the determination of the polarizability as a measure of biomass in disposable bioreactors, a contactless measurement method is essential to avoid any contamination of the sample introduced by the sensor. One promising approach is a sensing concept based on a differential transformer [1-3]. In this work, we present a new integrated differential transformer structured on a single printed circuit board (PCB). The differential transformer is characterized with sodium chloride solutions and compared to a highly sensitive but larger multi-layer differential transformer from an earlier work [4].
\end{abstract}

Keywords: differential transformer, contactless measurement, miniaturized PCB coils, inductive sensor, integrated differential transformer

\section{Introduction}

The basic setup of the differential transformer consists of three coils placed on a ferrite core as can be seen in Fig. 1.

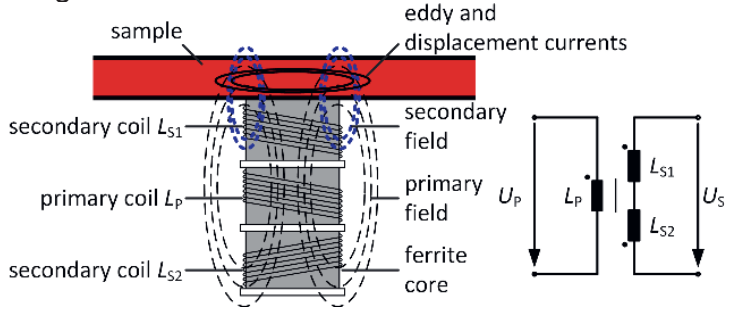

Fig. 1: Depiction of a differential transformer for the analysis of the electrical and dielectric properties of a sample (loaded) and its electrical equivalent circuit for the unloaded case.

Depending on the electrical conductivity $\kappa$ and the polarizability $\varepsilon^{\prime}$ of the sample, the primary magnetic field caused by the exited primary coil $L_{P}$ with an AC voltage $U_{P}$ induces eddy and displacement currents within the sample. Since the secondary coils $L_{s 1}$ and $L_{s 2}$ have the same inductance and are arranged symmetrically to $L_{p}$, but are differentially connected in series to each other, no secondary voltage $U_{s}$ can be measured due to the primary field for the unloaded (symmetric) differential transformer. This enables precise measurement of the weak induced currents within the sample. A detailed description of the measuring principle can be found in [5], where it is also shown that Us can be calculated according to Eq. 1 and separated into a real and an imaginary part. The imaginary part is indicated by the imaginary unit $j$. The real part depends on $\varepsilon^{\prime}$ and the imaginary part depends on $\kappa$ as well as the primitive losses $\varepsilon^{\prime \prime}$.

$U_{\mathrm{S}}=U_{\mathrm{P}} K\left(-\omega^{2} \varepsilon^{\prime}+\mathrm{j} \omega\left(\kappa-\omega \varepsilon^{\prime \prime}\right)\right)$
$K$ describes the magnetic coupling of the coils to each other as well as between the coils and the sample. $\omega$ is the angular frequency. Based on theoretical considerations, in [4] we have shown that the sensitivity of the differential transformer can be enhanced by optimizing the distance between the primary and secondary coils, as the difference in magnetic coupling of the two secondary coils to the sample should be as high as possible. However, at the same time, the magnetic coupling between the primary coil and the medium should not be reduced significantly by moving the coil too far away from the medium resulting in an optimal distance between the coils. These considerations could be proved by theoretical calculations as well as measurements.

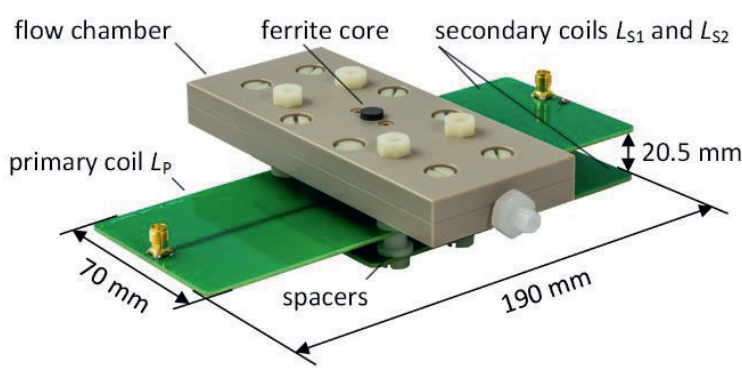

Fig. 2: Photograph of the previously used differential transformer with flow chamber [4]. $L_{s 1}$ and $L_{s 2}$ are differentially connected via electrical wires.

To obtain a differential transformer with adjustable distance between the coils, we used each coil on a separate printed circuit board (PCB). Each PCB has a size of $120 \mathrm{~mm}$ times $70 \mathrm{~mm}$. A photo of this previously used differential transformer is shown in Fig. 2. Spacers are used to adjust the distance between the PCB coils. The transformer in Fig. 2 uses $8 \mathrm{~mm}$ spacer corresponding to the optimal distance of this 
arrangement, resulting in a total height of the differential transformer excluding the measuring chamber of $20.5 \mathrm{~mm}$. The objective of this work is to optimize the differential transformer regarding its size to have a smaller transformer improving usability, even if this is accompanied by an inevitable reduction of sensitivity. Therefore, we present a new integrated differential transformer, where all coils are located on a single PCB.

\section{Integrated Differential Transformer Structured on a Single Printed Circuit Board}

Fig. 3 shows the side view of the new differential transformer structured on a single PCB (a) as well as the top view (b).

(a)

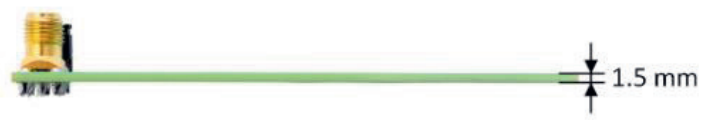

(b)

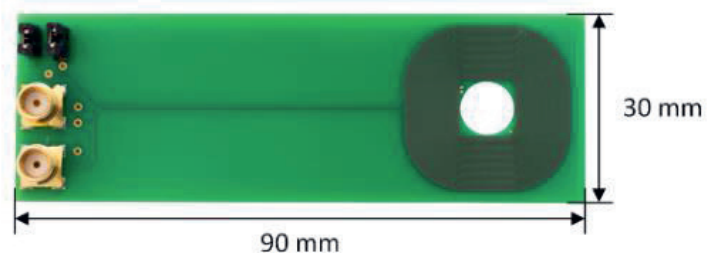

Fig 3: Photograph of the new differential transformer structured on a single PCB in side view (a) and top view (b).

All coils were realized on one $90 \mathrm{~mm}$ times $30 \mathrm{~mm}$ multi-layer PCB, giving the differential transformer an overall height of only $1.5 \mathrm{~mm}$. In total, the PCB has six layers. Each layer has a distance of $0.3 \mathrm{~mm}$ to the next layer. The secondary coils $L_{s 1}$ and $L_{s 2}$ are realized on the two top and two bottom layers respectively. The maximum outer radius of $L s_{1}$ and $L s_{2}$ are $14.5 \mathrm{~mm}$. Each secondary coil has a total number of turns of 70 and an inductance of $102 \mu \mathrm{H}$ (without ferrite core). The secondary inductances are thus significantly lower compared to the previously used differential transformer with $L_{s 1}$ and $L_{s 2}$ of $23.8 \mathrm{mH}$, representing a disadvantage in terms of sensitivity [4]. However, an advantage of this lower inductance is the higher resonant frequency of $2 \mathrm{MHz}$ compared to the secondary coil of the previously used differential transformer, operating at $155 \mathrm{kHz}$. This has a positive effect on sensitivity, as it increases with higher frequency [4]. However, to ensure that the coil still has an inductive characteristic, the measuring frequency should be below the resonance frequency. Here, we choose a frequency of $1 \mathrm{MHz}$. The primary coil with an outer radius of $9.5 \mathrm{~mm}$ and 30 turns, resulting in an inductance of $L_{P}$ is $17 \mu \mathrm{H}$ (without ferrite core) is located on the two middle layers. The differentially connected secondary coils as well as the primary coil can be easily connected via SMA connectors. For testing purposes, the differential connection of $L_{s 1}$ and $L_{s 2}$ is made via two jumpers, so that the connection can be opened to determine the inductance of each individual coil. A ferrite core with a diameter of $8 \mathrm{~mm}$ can be inserted through the center hole of the coils and hence improving the inductive coupling between the coils and the sample. Here we use a core with a relative permeability of 300 .

\section{Experimental Investigation}

In order to test the integrated differential transformer, it simply replaces the previously used differential transformer shown in Fig. 2. Using a peristaltic pump, a synthetic test solution consisting of deionized water and different concentrations of sodium chloride $(\mathrm{NaCl})$ flows through the flow chamber. The conductivity of the solution can be influenced by the $\mathrm{NaCl}$ concentration, affecting the imaginary part of the output voltage $U_{s}$. Fig. 4 shows the imaginary part of $U_{s}$ versus the $\mathrm{NaCl}$ concentration. It can be seen that the imaginary part of $U_{S}$ depends linearly on the $\mathrm{NaCl}$ concentration within this concentration range. This concentration range represents the clinical relevant pathological concentration range in blood serum. The sensitivity $S$ of the integrated differential transformer was determined by the slope of the linear regression with a coefficient of determination $\mathrm{R}^{2}$ of 0.99 and is about $11 \mathrm{mV} / \mathrm{mol} / \mathrm{L}$ at an excitation voltage $U_{P}$ of $1 V_{P P}$ peak to peak and a frequency of $1 \mathrm{MHz}$. As expected, the previously used differential transformer shown in Fig. 2 has a higher sensitivity of $192 \mathrm{mV} / \mathrm{mol} / \mathrm{L}$. Nevertheless, the main objective was to miniaturize and simplify the setup robust enough for everyday clinical use.

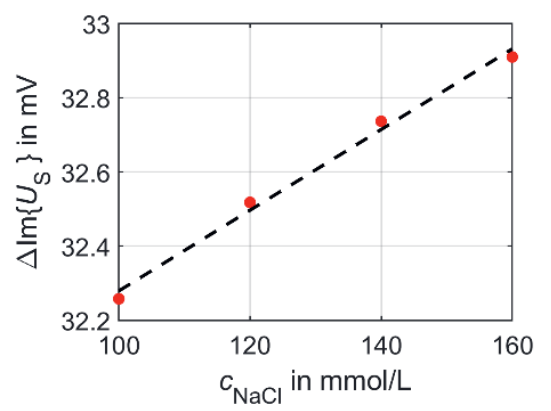

Fig 4: Imaginary part of the measuring voltage $U_{S}$ vs. the $\mathrm{NaCl}$ concentration and the linear regression $\left(R^{2}=0.99\right)$ with a sensitivity $S$ of about $11 \mathrm{mV} / \mathrm{mol} / \mathrm{L}$.

\section{Conclusion}

In this work, we have presented a new miniaturized differential transformer structured on a single PCB. This transformer was characterized using synthetic sodium chloride solutions at a concentration range from $100 \mathrm{mmol} / \mathrm{L}$ to $160 \mathrm{mmol} / \mathrm{L}$. Compared to the previously used much larger differential transformer the sensitivity is reduced. Nevertheless, this very compact, easy-to-use and robust design suitable for everyday clinical use has a sensitivity of $11 \mathrm{mV} / \mathrm{mol} / \mathrm{L}$ with very good linearity.

\section{References}

[1] M. Allers et al., Differential Inductive Sensor for Continuous Non-Invasive Cell Growth Monitoring in Disposable Bioreactors, Proceedings 1 (2017) 518.

[2] M. Berger et al., A Differential Transformer for Noninvasive Continuous Sodium Monitoring During Dialysis Treatment: 2019 IEEE SENSORS, pp. 1-4.

[3] M. Berger et al., Continuous Non-Invasive Sodium Monitoring in Extracorporeal Circuits: 17th International Meeting on Chemical - IMCS, pp. 683-684.

[4] M. Berger et al., Contactless and continuous sodium concentration monitoring during continuous renal replacement therapy, Sensors and Actuators B: Chemical $320(2020) 128372$.

[5] T. Reinecke, et al., Continuous noninvasive monitoring of cell growth in disposable bioreactors, Sensors and Actuators B: Chemical 251 (2017) 1009-1017. 